\title{
Ovarian cancer variant rs2072590 is associated with HOXD1 and HOXD3 gene expression
}

\author{
Liyuan Guo ${ }^{1, *}$, Yan Peng ${ }^{2, *}$, Lei Sun ${ }^{3, *}$, Xia Han ${ }^{4}$, Juan $\mathrm{Xu}^{4}$ and Dongwei Mao ${ }^{4}$ \\ ${ }^{1}$ Department of Gynecological Oncology, Cancer Hospital of Harbin Medical University, Harbin, China \\ ${ }^{2}$ Disease Prevention Center, First Affiliated Hospital, Heilongjiang University of Chinese Medicine, Harbin, China \\ ${ }^{3}$ Department of Gynecology and Obstetrics, The Fourth Hospital of Harbin Medical University, Harbin, China \\ ${ }^{4}$ Shenzhen Hospital of Guangzhou University of Chinese Medicine, Shenzhen, China \\ *These authors contributed equally to this work
}

Correspondence to: Dongwei Mao, email: maodongwei2017@126.com

Keywords: ovarian cancer, genome-wide association study, gene expression

Received: July 17, 2017 Accepted: September 21, $2017 \quad$ Published: October 13, 2017

Copyright: Guo et al. This is an open-access article distributed under the terms of the Creative Commons Attribution License 3.0 (CC BY 3.0), which permits unrestricted use, distribution, and reproduction in any medium, provided the original author and source are credited.

\section{ABSTRACT}

Ovarian cancer $(\mathrm{OC})$ is a common cancer in women and the leading cause of deaths from gynaecological malignancies in the world. In addition to the candidate gene approach to identify OC susceptibility genes, the genome-wide association study (GWAS) methods have reported new variants that are associated with OC risk. The minor allele of rs2072590 at 2q31 was associated with an increased OC risk, and was primarily significant for serous subtype. The OC risk-associated SNP rs2072590 lies in non-coding DNA downstream of HOXD3 and upstream of HOXD1, and it tags SNPs in the HOXD3 3' UTR. We think that the non-coding rs2072590 variant may contribute to OC susceptibility by regulating the gene expression of HOXD1 and HOXD3. In order to investigate this association, we performed a bioinformatics analysis by a functional annotation of rs2072590 variant using RegulomeDB (version 1.1), HaploReg (version 4.1), and PhenoScanner (version 1.1). Using HaploReg, we identified 19 genetic variants tagged by rs2072590 variant with with $r^{2}>=0.8$. Using RegulomeDB, we identified that three genetic variants are likely to affect TF binding + any motif + DNase Footprint + DNase peak. Other genetic variants are likely to affect TF binding + DNase peak. Using PhenoScanner (version 1.1), we identified that these 19 genetic variants could significantly regulate the expression of nearby genes, especially the HOXD1 and HOXD3 in human ovary tissue.

\section{INTRODUCTION}

Ovarian cancer $(\mathrm{OC})$ is a common cancer in women and the leading cause of deaths from gynaecological malignancies in the world [1]. Like other human complex diseases, OC is caused by the combination of genetic variants and environmental factors, including the familial BRCA1 and BRCA2 mutations and common genetic variants of lower penetrance [1]. In addition to the candidate gene approach to identify OC susceptibility genes, the genome-wide association study (GWAS) methods have also reported new variants that are associated with OC risk [1].

However, the exact genetic mechanisms for these OC susceptibility variants are still unclear [2]. It is reported that the potential associations between gene expression and OC risk alleles may connect risk variants to their putative target genes/transcripts and biological pathways [2]. The minor allele of rs2072590 at 2q31 was associated with an increased $\mathrm{OC}$ risk $(\mathrm{OR}=1.16$, $95 \%$ CI $\left.1.12-1.21, p=4.5 \times 10^{-14}\right)$, and was primarily significant for serous subtype $(\mathrm{OR}=1.20,95 \% \mathrm{CI}$ $\left.1.14-1.25, p=3.8 \times 10^{-14}\right)$ [3]. The $2 \mathrm{q} 31$ locus contains a family of homeobox (HOX) genes involved in regulating embryogenesis and organogenesis [3]. Altered expression of HOX genes has been reported in many cancers [3]. The OC risk-associated SNP rs2072590 lies in non-coding DNA downstream of $H O X D 3$ and upstream of $H O X D 1$, and it tags SNPs in the HOXD3 3' UTR [3]. 
We think that the non-coding rs2072590 variant may contribute to OC susceptibility by regulating the gene expression of HOXD1 and HOXD3. In order to investigate this association, we conducted a functional annotation of rs2072590 variant using RegulomeDB (version 1.1) [4], HaploReg (version 4.1) [5], and PhenoScanner (version 1.1) [6].

\section{RESULTS}

\section{LD analysis using HaploReg}

Using the LD information from the 1000 Genomes Project $(E U R)$, we got 19 genetic variants tagged by rs2072590 variant with with $\mathrm{r}^{2}>=0.8$. These 19 genetic variants are located around the HOXD4, HOXD3, AC009336.24 and HOXD-AS1. Here, we give the detailed information including the LD information about these variants in Table 1.

\section{Functional annotation using RegulomeDB}

RegulomeDB was used to annotate these 19 genetic variants with known and predicted regulatory elements. The results showed that three genetic variants including rs1562315, rs2551802 and rs6433571 likely to affect TF binding + any motif + DNase Footprint + DNase peak, as described in Table 2. Other genetic variants are likely to affect TF binding + DNase peak. More detailed results are described in Table 2.

\section{Functional annotation using PhenoScanner}

Using PhenoScanner (version 1.1), we identified that these 19 genetic variants could significantly regulate the expression of nearby genes including HOXDAS1, HOXD3, HOXD1, HOXD4, ATP5G3, HOXD9, HOXD11, KIAA1715, MTX2, LINC01116, HOXDAS2, HOXD8, and HOXD10 in 32 human tissues. These tissues include Adipose subcutaneous, Adipose visceral omentum, Artery tibial, Brain cerebellar hemisphere, Brain hippocampus, Brain nucleus accumbens basal ganglia, Brain putamen basal ganglia, Breast mammary tissue, Cells transformed fibroblasts, Colon sigmoid, Colon transverse, Esophagus gastroesophageal junction, Esophagus mucosa. Esophagus muscularis, Heart atrial appendage, Lung, Lymphoblastoid cell lines, Muscle skeletal, Nerve tibial, Ovary, Pancreas, Peripheral blood, Skin, Skin not sun exposed suprapubic, Skin sun exposed lower leg, Small intestine terminal ileum, Spleen, Stomach, Testis, Thyroid, Uterus and Whole blood. Interestingly, these genetic variants could significantly regulate the gene expression of HOXD1 and HOXD3 in human ovary tissue, as described in Table 3. More detailed results in 32 human tissues are described in Supplementary Table 1.

\section{DISCUSSION}

Overall, the GWAS methods have reported new variants that are associated with OC risk [1]. However, the exact genetic mechanisms for these OC susceptibility variants are still unclear [2]. Evidence shows that the potential associations between gene expression and OC risk alleles may connect risk variants to their putative target genes/transcripts and biological pathways [2]. Zhao et al. selected seven OC risk variants including rs3814113 on 9p22, rs2072590 on 2q31, rs2665390 on 3q25, rs 10088218, rs 1516982, rs10098821 on $8 \mathrm{q} 24$, and rs2363956 on 19p13 [2]. They evaluated the associations between gene expression and OC risk alleles using the whole genome mRNA expression data in 121 lymphoblastoid cell lines from 74 non-related familial ovarian cancer patients, and 47 non-cancer unrelated family controls [2]. They identified two cis-associations between $r s 10098821$ and $c-M y c$, and rs2072590 and HS. 565379.

The OC risk-associated SNP rs2072590 lies in non-coding DNA downstream of $H O X D 3$ and upstream of HOXD1, and it tags SNPs in the HOXD3 3' UTR [3]. However, Zhao et al. did not report any significant association between rs2072590 and HOXD1 or HOXD3. We think that the non-coding rs2072590 variant may contribute to $\mathrm{OC}$ susceptibility by regulating the gene expression of $H O X D 1$ and $H O X D 3$. Here, we conducted a functional annotation of rs2072590 variant using RegulomeDB (version 1.1) [4], HaploReg (version 4.1) [5], and PhenoScanner (version 1.1) [6].

Using HaploReg, we identified 19 genetic variants tagged by rs2072590 variant with with $r^{2}>=0.8$. Using RegulomeDB, we identified that three genetic variants are likely to affect TF binding + any motif + DNase Footprint + DNase peak. Other genetic variants are likely to affect TF binding + DNase peak. Using PhenoScanner (version 1.1), we identified that these 19 genetic variants could significantly regulate the expression of nearby genes, especially the HOXD1 and HOXD3 in human ovary tissue.

In addition to the $\mathrm{OC}$, some other comprehensive functional annotation of human complex diseases have also been conducted including colorectal cancer $[7,8]$, prostate cancer [9-11], breast cancer [12], multiple sclerosis [13], and Alzheimer's disease [14]. Collectively, we think that our results provide further insight into the genetic architecture of inherited susceptibility to OC, as did in previous studies [7-14].

\section{MATERIALS AND METHODS}

\section{LD analysis using HaploReg}

HaploReg is a tool for exploring annotations of the noncoding genome at variants on haplotype blocks 
Table 1: rs2072590 and variants with $r^{2}>=0.8$

\begin{tabular}{|c|c|c|c|c|c|c|c|c|}
\hline SNP & chromosome & pos (hg38) & $\operatorname{LD}\left(r^{2}\right)$ & LD (D') & Ref & Alt & Gene & Functional annotation \\
\hline rs4972504 & 2 & 176153998 & 0.89 & 0.98 & $\mathrm{~T}$ & $\mathrm{C}$ & HOXD4 & \\
\hline rs 2551802 & 2 & 176157430 & 0.85 & 0.93 & $\mathrm{C}$ & $\mathrm{G}$ & HOXD3 & \\
\hline rs 2252895 & 2 & 176159192 & 0.96 & 0.98 & A & G & HOXD3 & \\
\hline rs2252894 & 2 & 176159194 & 0.88 & 0.96 & G & $\mathrm{C}$ & HOXD3 & \\
\hline rs 2857538 & 2 & 176159533 & 0.98 & 1 & $\mathrm{C}$ & $\mathrm{T}$ & HOXD3 & \\
\hline rs 2857540 & 2 & 176161970 & 0.98 & 0.99 & G & $\mathrm{T}$ & HOXD3 & \\
\hline rs2113559 & 2 & 176166371 & 0.97 & 0.99 & A & G & HOXD3 & intronic \\
\hline rs 717852 & 2 & 176166895 & 0.98 & 1 & $\mathrm{C}$ & $\mathrm{T}$ & HOXD3 & intronic \\
\hline rs2249131 & 2 & 176167367 & 0.98 & 1 & $\mathrm{C}$ & $\mathrm{T}$ & HOXD3 & intronic \\
\hline rs 2857532 & 2 & 176168555 & 0.98 & 1 & A & G & HOXD3 & intronic \\
\hline rs1051929 & 2 & 176172026 & 1 & 1 & $\mathrm{~T}$ & $\mathrm{C}$ & HOXD3 & synonymous \\
\hline rs 711830 & 2 & 176172583 & 1 & 1 & $\mathrm{~A}$ & G & HOXD3 & 3'-UTR \\
\hline rs 1318778 & 2 & 176173103 & 1 & 1 & $\mathrm{C}$ & G & HOXD3 & \\
\hline rs 1549334 & 2 & 176174469 & 1 & 1 & G & A & HOXD3 & \\
\hline rs6433571 & 2 & 176174850 & 0.98 & 1 & G & $\mathrm{T}$ & HOXD3 & \\
\hline rs 2072590 & 2 & 176177905 & 1 & 1 & A & $\mathrm{C}$ & AC009336.24 & intronic \\
\hline rs6755766 & 2 & 176178477 & 0.96 & 1 & $\mathrm{~T}$ & $\mathrm{C}$ & AC009336.24 & intronic \\
\hline rs6755777 & 2 & 176178498 & 0.99 & 1 & $\mathrm{~T}$ & G & AC009336.24 & intronic \\
\hline rs 1562315 & 2 & 176180754 & 0.98 & 1 & $\mathrm{~T}$ & A & HOXD-AS1 & intronic \\
\hline
\end{tabular}

AFR, African samples; AMR, Ad Mixed American samples; ASN, East Asian samples; EUR, European samples; LD, linkage disequilibrium; SNP, single nucleotide polymorphism; Ref = reference allele; Alt = altered allele.

\section{Table 2: Functional annotation results using RegulomeDB}

\begin{tabular}{|c|c|c|c|c|c|}
\hline SNP & chromosome & pos (hg38) & Ref & Alt & Regulome DB Score \\
\hline rs 1562315 & 2 & 176180754 & $\mathrm{~T}$ & A & $2 \mathrm{~b}$ \\
\hline rs2551802 & 2 & 176157430 & $\mathrm{C}$ & G & $2 b$ \\
\hline rs6433571 & 2 & 176174850 & G & $\mathrm{T}$ & $2 b$ \\
\hline rs 1051929 & 2 & 176172026 & $\mathrm{~T}$ & $\mathrm{C}$ & 4 \\
\hline rs 1318778 & 2 & 176173103 & $\mathrm{C}$ & G & 4 \\
\hline rs 1549334 & 2 & 176174469 & G & A & 4 \\
\hline rs2072590 & 2 & 176177905 & A & $\mathrm{C}$ & 4 \\
\hline rs2249131 & 2 & 176167367 & $\mathrm{C}$ & $\mathrm{T}$ & 4 \\
\hline rs2252894 & 2 & 176159194 & G & $\mathrm{C}$ & 4 \\
\hline rs2252895 & 2 & 176159192 & A & G & 4 \\
\hline rs 2857538 & 2 & 176159533 & $\mathrm{C}$ & $\mathrm{T}$ & 4 \\
\hline rs 2857540 & 2 & 176161970 & G & $\mathrm{T}$ & 4 \\
\hline rs6755766 & 2 & 176178477 & $\mathrm{~T}$ & $\mathrm{C}$ & 4 \\
\hline rs 6755777 & 2 & 176178498 & $\mathrm{~T}$ & G & 4 \\
\hline rs711830 & 2 & 176172583 & A & G & 4 \\
\hline rs2113559 & 2 & 176166371 & A & G & 5 \\
\hline rs2857532 & 2 & 176168555 & A & G & 5 \\
\hline rs4972504 & 2 & 176153998 & $\mathrm{~T}$ & $\mathrm{C}$ & 5 \\
\hline rs717852 & 2 & 176166895 & $\mathrm{C}$ & $\mathrm{T}$ & 5 \\
\hline
\end{tabular}

1a, eQTL + TF binding + matched TF motif + matched DNase Footprint + DNase peak; 1b, eQTL + TF binding + any motif + DNase Footprint + DNase peak; 1c, eQTL + TF binding + matched TF motif + DNase peak; 1d, eQTL + TF binding + any motif + DNase peak; 1e, eQTL + TF binding + matched TF motif; 1f, eQTL + TF binding / DNase peak; 2a, TF binding + matched TF motif + matched DNase Footprint + DNase peak; $2 b$, TF binding + any motif + DNase Footprint + DNase peak; 2c, TF binding + matched TF motif + DNase peak; 3a, TF binding + any motif + DNase peak; 3b, TF binding + matched TF motif; 4, TF binding + DNase peak; 5, TF binding or DNase peak; 6 , other. 
Table 3: 19 genetic variants and gene expression in human ovary tissue

\begin{tabular}{|c|c|c|c|c|c|c|c|c|c|c|}
\hline SNP & Pos (hg19) & Alleles & Tissue & Gene & Ensembl & $N$ & Effect Allele & Beta & SE & $P$ \\
\hline rs1051929 & chr2:177036754 & $\mathrm{T} / \mathrm{C}$ & Ovary & HOXD1 & ENSG00000128645.11 & 85 & $\mathrm{~T}$ & 0.4891 & 0.1384 & 0.000765 \\
\hline rs1051929 & $\operatorname{chr} 2: 177036754$ & $\mathrm{~T} / \mathrm{C}$ & Ovary & HOXD3 & ENSG00000128652.7 & 85 & $\mathrm{~T}$ & 0.5701 & 0.1525 & 0.000396 \\
\hline rs1318778 & chr2:177037831 & $\mathrm{C} / \mathrm{G}$ & Ovary & HOXD1 & ENSG00000128645.11 & 85 & $\mathrm{C}$ & 0.4921 & 0.1367 & 0.000623 \\
\hline rs1318778 & $\operatorname{chr} 2: 177037831$ & $\mathrm{C} / \mathrm{G}$ & Ovary & HOXD3 & ENSG00000128652.7 & 85 & $\mathrm{C}$ & 0.5721 & 0.1507 & 0.000328 \\
\hline rs1549334 & $\operatorname{chr} 2: 177039197$ & $\mathrm{G} / \mathrm{A}$ & Ovary & HOXD1 & ENSG00000128645.11 & 85 & G & 0.4921 & 0.1367 & 0.000623 \\
\hline rs1549334 & $\operatorname{chr} 2: 177039197$ & $\mathrm{G} / \mathrm{A}$ & Ovary & HOXD3 & ENSG00000128652.7 & 85 & G & 0.5721 & 0.1507 & 0.000328 \\
\hline rs1562315 & $\operatorname{chr} 2: 177045482$ & $\mathrm{~T} / \mathrm{A}$ & Ovary & HOXD1 & ENSG00000128645.11 & 85 & $\mathrm{~T}$ & 0.4404 & 0.1334 & 0.00158 \\
\hline rs 1562315 & $\operatorname{chr} 2: 177045482$ & $\mathrm{~T} / \mathrm{A}$ & Ovary & HOXD3 & ENSG00000128652.7 & 85 & $\mathrm{~T}$ & 0.5172 & 0.147 & 0.000804 \\
\hline rs2072590 & $\operatorname{chr} 2: 177042633$ & $\mathrm{C} / \mathrm{A}$ & Ovary & HOXD1 & ENSG00000128645.11 & 85 & $\mathrm{C}$ & -0.4923 & 0.1367 & 0.00062 \\
\hline rs2072590 & $\operatorname{chr} 2: 177042633$ & $\mathrm{C} / \mathrm{A}$ & Ovary & HOXD3 & ENSG00000128652.7 & 85 & $\mathrm{C}$ & -0.5724 & 0.1507 & 0.000327 \\
\hline rs2113559 & chr2:177031099 & $\mathrm{G} / \mathrm{A}$ & Ovary & HOXD1 & ENSG00000128645.11 & 85 & G & -0.4623 & 0.1444 & 0.002132 \\
\hline rs2113559 & chr2:177031099 & $\mathrm{G} / \mathrm{A}$ & Ovary & HOXD3 & ENSG00000128652.7 & 85 & G & -0.5461 & 0.159 & 0.001047 \\
\hline rs2249131 & $\operatorname{chr} 2: 177032095$ & $\mathrm{C} / \mathrm{T}$ & Ovary & HOXD1 & ENSG00000128645.11 & 85 & $\mathrm{C}$ & 0.4629 & 0.1441 & 0.002068 \\
\hline rs2249131 & $\operatorname{chr} 2: 177032095$ & $\mathrm{C} / \mathrm{T}$ & Ovary & HOXD3 & ENSG00000128652.7 & 85 & $\mathrm{C}$ & 0.5464 & 0.1587 & 0.001019 \\
\hline rs2252894 & chr2:177023922 & $\mathrm{C} / \mathrm{G}$ & Ovary & HOXD1 & ENSG00000128645.11 & 85 & $\mathrm{C}$ & -0.3923 & 0.1462 & 0.009254 \\
\hline rs2252895 & chr2:177023920 & $\mathrm{A} / \mathrm{G}$ & Ovary & HOXD1 & ENSG00000128645.11 & 85 & A & 0.4738 & 0.1439 & 0.001618 \\
\hline rs2252895 & $\operatorname{chr} 2: 177023920$ & $\mathrm{~A} / \mathrm{G}$ & Ovary & HOXD3 & ENSG00000128652.7 & 85 & A & 0.5187 & 0.1605 & 0.001948 \\
\hline rs 2551802 & $\operatorname{chr} 2: 177022158$ & $\mathrm{C} / \mathrm{G}$ & Ovary & HOXD1 & ENSG00000128645.11 & 85 & $\mathrm{C}$ & 0.4433 & 0.1374 & 0.001984 \\
\hline rs2551802 & chr2:177022158 & $\mathrm{C} / \mathrm{G}$ & Ovary & HOXD3 & ENSG00000128652.7 & 85 & $\mathrm{C}$ & 0.5068 & 0.1522 & 0.001447 \\
\hline rs 2857532 & chr2:177033283 & $\mathrm{A} / \mathrm{G}$ & Ovary & HOXD1 & ENSG00000128645.11 & 85 & A & 0.4629 & 0.1441 & 0.002068 \\
\hline rs 2857532 & $\operatorname{chr} 2: 177033283$ & $\mathrm{~A} / \mathrm{G}$ & Ovary & HOXD3 & ENSG00000128652.7 & 85 & $\mathrm{~A}$ & 0.5464 & 0.1587 & 0.001019 \\
\hline rs2857538 & $\operatorname{chr} 2: 177024261$ & $\mathrm{C} / \mathrm{T}$ & Ovary & HOXD1 & ENSG00000128645.11 & 85 & $\mathrm{C}$ & 0.4715 & 0.1435 & 0.001652 \\
\hline rs 2857538 & chr2:177024261 & $\mathrm{C} / \mathrm{T}$ & Ovary & HOXD3 & ENSG00000128652.7 & 85 & $\mathrm{C}$ & 0.532 & 0.1593 & 0.001399 \\
\hline rs2857540 & chr2:177026698 & $\mathrm{G} / \mathrm{T}$ & Ovary & HOXD1 & ENSG00000128645.11 & 85 & G & 0.4197 & 0.15 & 0.006799 \\
\hline rs 2857540 & chr2:177026698 & $\mathrm{G} / \mathrm{T}$ & Ovary & HOXD3 & ENSG00000128652.7 & 85 & G & 0.5023 & 0.1653 & 0.003439 \\
\hline rs 4972504 & $\operatorname{chr} 2: 177018726$ & $\mathrm{C} / \mathrm{T}$ & Ovary & HOXD1 & ENSG00000128645.11 & 85 & $\mathrm{C}$ & -0.4658 & 0.1416 & 0.001635 \\
\hline rs4972504 & chr2:177018726 & $\mathrm{C} / \mathrm{T}$ & Ovary & HOXD3 & ENSG00000128652.7 & 85 & $\mathrm{C}$ & -0.5097 & 0.158 & 0.001977 \\
\hline rs6433571 & chr2:177039578 & $\mathrm{G} / \mathrm{T}$ & Ovary & HOXD1 & ENSG00000128645.11 & 85 & G & 0.4584 & 0.1353 & 0.00121 \\
\hline rs6433571 & chr2:177039578 & $\mathrm{G} / \mathrm{T}$ & Ovary & HOXD3 & ENSG00000128652.7 & 85 & G & 0.5254 & 0.1497 & 0.000827 \\
\hline rs6755766 & chr2:177043205 & $\mathrm{C} / \mathrm{T}$ & Ovary & HOXD1 & ENSG00000128645.11 & 85 & $\mathrm{C}$ & -0.4481 & 0.1368 & 0.001702 \\
\hline rs6755766 & chr2:177043205 & $\mathrm{C} / \mathrm{T}$ & Ovary & HOXD3 & ENSG00000128652.7 & 85 & $\mathrm{C}$ & -0.5348 & 0.1502 & 0.000705 \\
\hline rs6755777 & chr2:177043226 & $\mathrm{G} / \mathrm{T}$ & Ovary & HOXD1 & ENSG00000128645.11 & 85 & G & -0.4405 & 0.1334 & 0.001577 \\
\hline rs6755777 & chr2:177043226 & $\mathrm{G} / \mathrm{T}$ & Ovary & HOXD3 & ENSG00000128652.7 & 85 & G & -0.5171 & 0.147 & 0.000807 \\
\hline rs711830 & chr2:177037311 & $\mathrm{G} / \mathrm{A}$ & Ovary & HOXD1 & ENSG00000128645.11 & 85 & G & -0.4921 & 0.1367 & 0.000623 \\
\hline rs711830 & chr2:177037311 & $\mathrm{G} / \mathrm{A}$ & Ovary & HOXD3 & ENSG00000128652.7 & 85 & G & -0.5721 & 0.1507 & 0.000328 \\
\hline rs717852 & chr2:177031623 & $\mathrm{T} / \mathrm{C}$ & Ovary & HOXD1 & ENSG00000128645.11 & 85 & $\mathrm{~T}$ & -0.4629 & 0.1441 & 0.002068 \\
\hline rs717852 & chr2:177031623 & $\mathrm{T} / \mathrm{C}$ & Ovary & HOXD3 & ENSG00000128652.7 & 85 & $\mathrm{~T}$ & -0.5464 & 0.1587 & 0.001019 \\
\hline
\end{tabular}

[5]. HaploReg includes LD information from the 1000 Genomes Project, chromatin state and protein binding annotation from the Roadmap Epigenomics and the Encyclopedia of DNA Elements (ENCODE) projects, sequence conservation across mammals, the effect of SNPs on regulatory motifs, and the effect of SNPs on gene expression from eQTL studies [5]. We used HaploReg (version 4.1) to identify the rs2072590 tagged variants 
using the LD information from the 1000 Genomes Project $(E U R)$ with $\mathrm{r}^{2}>=0.8[5]$.

\section{Functional annotation using RegulomeDB}

RegulomeDB (version 1.1) is a database that annotates SNPs with known and predicted regulatory elements in the intergenic regions of the human genome [4]. Known and predicted regulatory DNA elements include regions of DNAase hypersensitivity, binding sites of transcription factors, and promoter regions that have been biochemically characterized to regulation transcription [4]. RegulomeDB (version 1.1) includes the public datasets from Gene Expression Omnibus (GEO), the ENCODE project, and published literature [4].

\section{Functional annotation using PhenoScanner}

PhenoScanner (version 1.1) is a curated database holding publicly available results from large-scale GWAS [6]. The motivation for creating this tool is to facilitate "phenome scans", the cross-referencing of genetic variants with a broad range of phenotypes, to help aid the understanding of disease pathways and biology [6]. The catalogue currently contains nearly 3 billion associations and over 10 million unique SNPs [6]. The results are aligned across traits to the same effect and non-effect alleles for each SNP [6].

\section{CONFLICTS OF INTEREST}

The authors declare no competing financial interests.

\section{REFERENCES}

1. Chen K, Ma H, Li L, Zang R, Wang C, Song F, Shi T, Yu D, Yang M, Xue W, Dai J, Li S, Zheng H, et al. Genomewide association study identifies new susceptibility loci for epithelial ovarian cancer in Han Chinese women. Nat Commun. 2014; 5:4682.

2. Zhao H, Shen J, Wang D, Guo Y, Gregory S, Medico L, Hu Q, Yan L, Odunsi K, Lele S, Liu S. Associations between gene expression variations and ovarian cancer risk alleles identified from genome wide association studies. PLoS One. 2012; 7:e47962.

3. Goode EL, Chenevix-Trench G, Song H, Ramus SJ, Notaridou M, Lawrenson K, Widschwendter M, Vierkant RA, Larson MC, Kjaer SK, Birrer MJ, Berchuck A, Schildkraut J, et al. A genome-wide association study identifies susceptibility loci for ovarian cancer at $2 \mathrm{q} 31$ and 8q24. Nat Genet. 2010; 42:874-9.
4. Boyle AP, Hong EL, Hariharan M, Cheng Y, Schaub MA, Kasowski M, Karczewski KJ, Park J, Hitz BC, Weng S, Cherry JM, Snyder M. Annotation of functional variation in personal genomes using RegulomeDB. Genome Res. 2012; $22: 1790-7$.

5. Ward LD, Kellis M. HaploReg v4: systematic mining of putative causal variants, cell types, regulators and target genes for human complex traits and disease. Nucleic Acids Res. 2016; 44:D877-81.

6. Staley JR, Blackshaw J, Kamat MA, Ellis S, Surendran P, Sun BB, Paul DS, Freitag D, Burgess S, Danesh J, Young R, Butterworth AS. PhenoScanner: a database of human genotype-phenotype associations. Bioinformatics. 2016; 32:3207-9.

7. Lu X, Cao M, Han S, Yang Y, Zhou J. Colorectal cancer risk genes are functionally enriched in regulatory pathways. Sci Rep. 2016; 6:25347.

8. Yao L, Tak YG, Berman BP, Farnham PJ. Functional annotation of colon cancer risk SNPs. Nat Commun. 2014; 5:5114.

9. Jiang J, Jia P, Shen B, Zhao Z. Top associated SNPs in prostate cancer are significantly enriched in cisexpression quantitative trait loci and at transcription factor binding sites. Oncotarget. 2014; 5:6168-77. https://doi. org/10.18632/oncotarget.2179.

10. Hazelett DJ, Rhie SK, Gaddis M, Yan C, Lakeland DL, Coetzee SG, Henderson BE, Noushmehr H, Cozen W, Kote-Jarai Z, Eeles RA, Easton DF, Haiman CA, et al. Comprehensive functional annotation of 77 prostate cancer risk loci. PLoS Genet. 2014; 10:e1004102.

11. Lu Y, Zhang Z, Yu H, Zheng SL, Isaacs WB, Xu J, Sun J. Functional annotation of risk loci identified through genome-wide association studies for prostate cancer. Prostate. 2011; 71:955-63.

12. Rhie SK, Coetzee SG, Noushmehr H, Yan C, Kim JM, Haiman CA, Coetzee GA. Comprehensive functional annotation of seventy-one breast cancer risk Loci. PLoS One. 2013; 8:e63925.

13. Disanto G, Kjetil Sandve G, Ricigliano VA, Pakpoor J, Berlanga-Taylor AJ, Handel AE, Kuhle J, Holden L, Watson CT, Giovannoni G, Handunnetthi L, Ramagopalan SV. DNase hypersensitive sites and association with multiple sclerosis. Hum Mol Genet. 2014; 23:942-8.

14. Jiang Q, Jin S, Jiang Y, Liao M, Feng R, Zhang L, Liu G, Hao J. Alzheimer's Disease Variants with the GenomeWide Significance are Significantly Enriched in Immune Pathways and Active in Immune Cells. Mol Neurobiol. 2017; 54:594-600. 